This item was submitted to Loughborough's Research Repository by the author.

Items in Figshare are protected by copyright, with all rights reserved, unless otherwise indicated.

\title{
Chaos in atmospheric-pressure plasma jets
}

PLEASE CITE THE PUBLISHED VERSION

http://dx.doi.org/10.1088/0963-0252/21/3/034008

\section{PUBLISHER}

(C) IOP Publishing Ltd

\section{VERSION}

AM (Accepted Manuscript)

\section{PUBLISHER STATEMENT}

This work is made available according to the conditions of the Creative Commons Attribution-NonCommercialNoDerivatives 4.0 International (CC BY-NC-ND 4.0) licence. Full details of this licence are available at: https://creativecommons.org/licenses/by-nc-nd/4.0/

\section{LICENCE}

CC BY-NC-ND 4.0

\section{REPOSITORY RECORD}

Walsh, James L., Felipe Iza, Natalia B. Janson, and Michael G. Kong. 2019. "Chaos in Atmospheric-pressure Plasma Jets". figshare. https://hdl.handle.net/2134/16397. 


\section{Chaos in atmospheric pressure plasma jets.}

J.L. Walsh ${ }^{1}$, F. Iza ${ }^{2}$, N.B. Janson ${ }^{3} \&$ M.G. Kong ${ }^{2}$

${ }^{1}$ Department of Electrical Engineering and Electronics, University of Liverpool, $L 69$ 3BX, UK

${ }^{2}$ School of Electronic and Electrical Engineering, Loughborough University, Loughborough, Leicestershire LE11 3TU, UK

${ }^{3}$ School of Mathematics, Loughborough University, Loughborough, Leicestershire LE11 3TU, UK

Abstract: We report detailed characterization of a low-temperature atmosphericpressure plasma jet that exhibits regimes of periodic, quasi-periodic and chaotic behaviors. Power spectra, phase portraits, stroboscopic section and bifurcation diagram of the discharge current combine to comprehensively demonstrate the existence of chaos, and this evidence is strengthened with a nonlinear dynamics analysis using two control parameters that maps out periodic, period-multiplication, and chaotic regimes over a wide range of the input voltage and gas flow rate. In addition, optical emission signatures of excited plasma species are used as the second and independent observable to demonstrate the presence of chaos and period-doubling in both the concentrations and composition of plasma species, suggesting a similar array of periodic, quasi-periodic and chaotic regimes in plasma chemistry. The presence of quasi-periodic and chaotic regimes in structurally unbounded low-temperature atmospheric plasmas not only is important as a fundamental scientific topic but also has interesting implications for their numerous applications. Chaos may be undesirable for industrial applications where cycle-tocycle reproducibility is important, yet for treatment of cell-containing materials including living tissues it may offer a novel route to combat some of the major challenges in medicine such as drug resistance. Chaos in low-temperature atmospheric plasmas and its effective control are likely to open up new vistas for medical technologies. 


\section{Introduction}

Low-temperature atmospheric-pressure plasma jets provide a unique opportunity to generate a non-equilibrium gas discharge that is rich in reactive chemical species yet is unbound by any electrode structure $[1,2]$. In addition to many industrial applications [3-5], such devices are finding increasing applications in the field of medicine; promising examples include prion inactivation [6], chronic wound treatment and cancer therapy $[7,8]$. Clearly, the use of plasma in medicine opens up new vistas of novel therapies and medical technologies-a breakthrough that could well become as important as that which was initiated by antibiotics [8]. Of the many systems proposed for the generation of stable atmospheric-pressure plasma, the dielectric barrier discharge (DBD) jet has become the configuration of choice for many researchers $[1,2]$. A typical plasma jet consists of a capillary formed from a dielectric material through which a noble gas flows. A metallic electrode, excited using $\mathrm{kHz}$ sinusoidal or short duration high-voltage pulses [9, 10], ionizes the flowing gas, resulting in a luminous plume that typically extends for several centimeters. A key advantage of the DBD jet configuration is that the reactive species generated in the capillary are effectively transported to a downstream sample where they are able to interact without compromising the stability of the device $[11,12]$. Depending on dissipated electric power, these atmospheric plasmas have been shown to operate in different modes including Townsend, glow and nonthermal arc modes, all modulated at the driving frequency [13]. Recently, however, it has been reported that atmospheric-pressure plasma jets can exhibit chaotic behavior under certain conditions [13-15]. From the standpoint of practical applications, chaotic behaviors of atmospheric plasmas raise questions of whether their interactions with a sample may become nonlinear with respect to time, particularly when the sample contains living organisms. Experimental evidence of 
chaos in atmospheric plasmas has so far relied overly on temporal features of a single observable [13- 15] and must be strengthened with detailed analysis of nonlinear dynamics of low-temperature atmospheric plasmas.

Chaos in gas discharges systems, other than low-temperature atmospheric plasmas, is known with its nonlinear characteristics comprehensively studied in various laboratory plasmas including low-pressure processing plasmas [16- 18] and high-pressure arcs $[19,20]$. However, in most cases, the plasmas studied are often undriven, i.e. the chaotic behavior arises from self-oscillation within steady-state (dc) plasma [16-21] although chaos in driven plasmas has also been reported [22]. In the case of atmospheric-pressure DBD jets, the discharge is generated using a fixed frequency; consequently the system can be considered to be driven. Although chaos in parallel-plate atmospheric DBDs have been proposed theoretically [23, 24], its experimental evidence has been reported in only three previous studies [13-15]. For chaos in atmospheric plasmas to be fully confirmed experimentally, it is important to employ a range of indicators with more than one observable [25]. Considering that high-pressure barrier discharges are used extensively in many materials processing, lighting and display applications [26- 28], it is surprising that few experimental studies on their nonlinear dynamic behaviors have been undertaken to date. This is therefore an important question in fundamental plasma physics with implication to downstream reaction chemistry of atmospheric plasma jets.

The paper is organized as follows. Section 2 provides details of the atmospheric-pressure plasma jet used in this study, including the description of the electrical and optical diagnostics employed. Section 3 illustrates how deterministic chaos in a plasma jet manifests itself by examining the impact of a single control parameter on a single variable being observed. This analysis is then extended further by introducing a second control parameter. Through the use of two control 
parameters, the dynamical behavior of the system is mapped onto the parameter plane, in which regions with qualitatively similar behaviors are outlined. Finally, the optical signatures of various excited states within the plasma are tracked with a high temporal resolution to provide an insight into both the underpinning physics of the discharge, as the system dynamics changes, and the discharge chemistry resulting from such changes. Section 4 concludes with a summary of the results.

\section{Experimental Setup}

The atmospheric plasma jet used in this study consists of a quartz tube $5 \mathrm{~cm}$ in length with a $1.6 \mathrm{~mm}$ inner diameter. A copper electrode was wrapped around the tube at a distance $1 \mathrm{~cm}$ from the open end, and a gas feed was connected at the opposite end. A high-voltage power source, comprising an Amplifier Research broadband amplifier and a high-voltage transformer, was connected to the wrapped electrode. A TTi TG2000 function generator was used to generate the driving sinusoidal signal to the amplifier, and the amplitude of this signal was used as a control parameter. Helium gas $(99.999 \%$ purity) at a nominal rate of 4 standard liters per minute (s/m) was ionized by the power source, and its flow rate was used as the second control parameter. The ionized gas exited the tube as a visible plume and struck a grounded electrode situated $1 \mathrm{~cm}$ away from the tube exit. Figure 1 provides a schematic representation of the experimental apparatus. Voltage measurements were made on the powered electrode using a Tektronix P6015A 75 MHz voltage probe, and current measurements were made with a Pearson 2877200 $\mathrm{MHz}$ current monitor. To provide an indicator of the underpinning reaction chemistry in the plasma, optical emission of excited helium at $706 \mathrm{~nm}(3 \mathrm{~s} 3 \mathrm{~S} \rightarrow 2 \mathrm{p} 3 \mathrm{P})$ and excited oxygen atoms at $777 \mathrm{~nm}(3 \mathrm{p} 5 \mathrm{~S} \rightarrow 3 \mathrm{~s} 5 \mathrm{P})$ were measured as a function of time using two Hamamatsu R1894 photomultiplier tubes (PMTs) with a 0.8 ns rise time and $4.2 \times 105$ gain. Each photomultiplier was used in conjunction with a band pass 
wavelength filter to allow the temporal profile of specific emission lines to be obtained. The filters have a $10 \mathrm{~nm}$ FWHM and were sourced from Thorlabs Ltd, UK. A Tektronix DPO 4104 1GHz oscilloscope was used to record electrical and PMT data, which was saved directly to a second PC via USB interface. All electrical and PMT signals were recorded using 100000 sample points and a sample time of $10 \mathrm{~ms}$. At a driving frequency of $45 \mathrm{kHz}$ this sampling equates to 450 voltage cycles with a resolution of 222 samples per cycle. To facilitate a tight control over experimental parameters, the high-voltage power source and gas flow controller were automated using a PC running MATLABß program.

\section{Results \& Discussion}

Possible chaos in the atmospheric plasma jet is studied in terms of its nonlinear dynamics initially with one electrical observable (i.e. the discharge current and its related electrical power dissipated in the plasma). This investigation employs firstly the input voltage as one single control parameter and then introduces the gas flow rate as the second control parameter, discussions of which are referred to as one-parameter analysis and two-parameter analysis, respectively. To relate the results of these nonlinear dynamics to downstream reaction chemistry that is central to applications of atmospheric-pressure plasma jets, optical emission signatures of excited helium and atomic oxygen are used as the second observable. Given that many neutral plasma species vary at a different timescale to electrons, it is uncertain whether the dynamics of reactive plasma species may be affected by their accumulation from one excitation cycle to the next and as a result may not attain characteristics of chaos. In other words, reaction chemistry may not necessarily be guaranteed even when chaos in the electron dynamics is confirmed for the same plasma. Therefore, the use of optical emission signatures as the second observable not only strengthens the case for chaos in electron dynamics, but also establishes 
whether this has any practical implications by examining existence of chaos in downstream reaction chemistry.

\subsection{One-parameter analysis}

Figures 2(a)-(c) highlight current and voltage traces of the plasma jet operating at an input voltage of $3.5 \mathrm{~V}, 5.8 \mathrm{~V}$ and $7.5 \mathrm{~V}$, respectively. Typically, barrier discharges exhibit a single discharge event every half cycle of the applied voltage, indicated by a single current spike [29], as is the case in figure 2(c). In figure 2(a), this pattern no longer holds true, with several half cycles of the applied voltages occurring with no obvious current spike being observed. In figure 2(b), a current spike is observed every half cycle of the applied voltage; however, both the magnitude and temporal position of the current spike vary from cycle to cycle. As the input voltage changes, a clear shift in the dynamics of the system is observed. To gain a further insight into the cases depicted in figures $2(a)-(c)$, the power spectral densities (spectra) are shown in figures 2(d)-(f), respectively. Each spectrum is obtained by calculating the squared amplitude of the Fourier transform of the discharge current, which was observed over 450 applied voltage cycles and averaged over 100 different realizations of the current. The ideal spectrum of a periodic process consists of peaks of zero width (delta peaks) at harmonics of the driving frequency. Therefore, figure 2(f) clearly indicates periodic behavior. The spectrum in figure 2(e) contains not only harmonics of the driving frequency, but also delta peaks at other frequencies, which is a characteristic of quasi-periodic behavior. It is known that the spectra of deterministically chaotic and of random processes are continuous functions of frequency [30]. While they may contain sharp peaks, these are imposed on a continuous background that does not disappear if the observation time increases. The spectrum shown in figure 2(d) has these features and is therefore an indication of the non-periodic nature of the current oscillations. The combination of 
figures 2(a) and (d) suggests that the behavior of the atmospheric plasma jet is likely to be chaotic at an input voltage of $3.5 \mathrm{~V}$. However, results contained in the temporal features of the electrical observable and its power spectra are not sufficiently conclusive to confirm the presence of deterministic chaos.

The difference between a chaotic and a periodic behavior of a system can be illustrated by a different approach borrowed from dynamical systems theory. It is assumed that an oscillating system can be mathematically described as a dynamical system. This means that at any time it has a state, defined as an ordered set of its variables (a state vector in the phase space), and there exists a rule, according to which the system goes from one state to the next (i.e. the evolution operator, often described as a collection of first order ordinary differential equations). The motion of the system can be graphically represented by the point in the phase space, which moves with time along a certain trajectory known as the phase trajectory. In practice, all oscillating systems are dissipative with an important property: starting from some initial conditions from a certain range, their motion is settled down to some repetitive, reproducible mode. This mode can be achieved from a broad range of initial conditions, and in the phase space it is represented by a geometrical object called the attractor. Periodic oscillations are represented by stable closed orbits (or limit cycles), whereas quasi-periodic oscillations are represented by toroidal orbits. However, chaotic oscillations are represented by the objects of complex shape and non-integer dimension known as chaotic attractors. Thus, one can distinguish between different types of the system behavior using phase portraits, which are in practice phase trajectories settled down on the attractor. The detailed structure of an attractor can be revealed by means of Poincare' section, a cross-section of the attractor by a certain surface that must cross (but not touch) all the phase 
trajectories [30]. A section of a limit cycle is depicted by one or several points, while the section of a chaotic attractor is typically a set with self-similar structure.

From a mathematical viewpoint, the plasma jet under study is a spatially extended system, in which currents and voltages depend not only on time, but also on space. Strictly speaking, the phase space of this system is formed by the full collection of space-dependent functions, which change in time as the system evolves, and the dimension of this system is infinitely large. It has been shown, however, that many infinite-dimensional dynamical systems can have attractors of finite dimension [31-33]. As often happens in laboratory experiments, not only is the explicit form of the evolution equations unknown to the researchers but also the full set of state variables is inaccessible. Typically, only a very limited number of experimentally accessible variables can be registered, often reduced to one or two. Fortunately, dynamical system theory allows one to visualize the dynamical behavior of such systems using the information available, by making use of a series of mathematical results [34-36]. Namely, it is possible to reconstruct the phase trajectory of the system from only a single registered variable by a popular technique called delay embedding [30]. Suppose one observes only the current $\mathrm{I}(\mathrm{t})$ at the driving electrode (i.e. the powered electrode). The state vector at time $\mathrm{t}$ in the $\mathrm{m}$ dimensional phase space is reconstructed as a vector whose coordinates are the values of $\mathrm{I}(\mathrm{t})$ taken at time moments separated by a certain delay $\mathrm{T}$, namely $(\mathrm{I}(\mathrm{t})$, $\mathrm{I}(\mathrm{t}+\mathrm{T}), \mathrm{I}(\mathrm{t}+2 \mathrm{~T}), \ldots, \mathrm{I}(\mathrm{t}+(\mathrm{m}-1) \mathrm{T}))$. The number $\mathrm{m}$ is the embedding dimension and depends on the dimension of the attractor in the original dynamical system. For visualization purposes, here we choose $\mathrm{m}=3$. The time delay $\mathrm{T}$ was determined by calculating the mutual information from the variables $\mathrm{I}(\mathrm{t})$ and $\mathrm{I}(\mathrm{t}+\mathrm{T})$ as a function of $\mathrm{T}$, and choosing its first minimum [37]. For all datasets studied, the value of $\mathrm{T}$ 
obtained by this technique was close to $4 \mu \mathrm{s}$ and was chosen for the reconstruction of all phase portraits.

Figures $3(\mathrm{a})-(\mathrm{c})$ show the phase portraits reconstructed in the threedimensional phase space for the plasma jet operating with an input voltage of $3.5 \mathrm{~V}$, $5.8 \mathrm{~V}$ and $7.5 \mathrm{~V}$, respectively. For each input voltage, the phase trajectory is shown during several hundred excitation cycles. In figures 3(b) and (c), closed loops (limit cycles) are shown whereas in figure 3(a) a set is shown that does not look similar either to a limit cycle (indicating periodic oscillations) or to a low-dimensional torus (indicating quasi-periodic oscillations). Consequently, this is likely to be a projection of a chaotic attractor into the three-dimensional space of figure $3(a)$. To obtain further evidence, we calculate an analog of the Poincare' section- a stroboscopic section, which is more convenient for systems perturbed periodically. This is obtained by collecting the points in the phase space at times $t_{0}, t_{0}+T_{R F}$, to $+2 T_{R F}, \ldots$, where $\mathrm{T}_{\mathrm{RF}}=22.2 \mu \mathrm{s}>\mathrm{T}=4 \mu \mathrm{s}$ is the period of the driving frequency at $45 \mathrm{kHz}$. Figures 3(d)-(f) show the stroboscopic sections calculated from the phase space reconstructions in figures 3(a)-(c), respectively. Figure 3(f) shows a set consisting of a single point, highlighting the periodicity of the respective oscillations. Figure 3(e) shows a set of four scattered points on the Poincare' section, thus indicating the presence of a so-called 'period-four' periodic oscillation, signifying that the phase trajectory makes four loops before closing on itself. Here its period is $4 P(P=T R F)$. This behavior might have occurred via a period-doubling bifurcation as the control parameters were changed $[23,24]$. On the other hand, figure $3(d)$ shows a complexlooking set of points, a strong argument for the existence of a chaotic attractor in the system.

Next, we produce a set of one-parameter bifurcation diagrams to characterize how the discharge dynamics evolve as a single control parameter is varied. A typical 
one-parameter bifurcation diagram, obtained when the input voltage is slowly increased from 2.5 to $10 \mathrm{~V}$, is displayed in figure $3(\mathrm{~g})$. The voltage intervals where the oscillations are regular (smooth branches) can be clearly distinguished from those at which oscillations are irregular (scatter plot). In the pre-breakdown regime of Vin $=2.5-3.4 \mathrm{~V}$, the measured current is simply the displacement current, and the system is periodic with a period of $1 \mathrm{P}$. Immediately after the breakdown, the bifurcation diagram resembles a scatter plot indicating chaotic oscillation, and this behavior continues until $3.54 \mathrm{~V}$, when the discharge becomes periodic with period $2 \mathrm{P}$. As input voltage is increased further, the discharge remains in a $2 \mathrm{P}$ state until 4.92 V, at which point the diagram appears to diverge into a set of closely scattered points, indicating the phase space reconstruction is that of a torus, suggesting quasiperiodic behavior. Between 5.5 and $6.2 \mathrm{~V}$ the diagram shows several period multiplications, and close inspection indicates that this is dominated by $2 \mathrm{P}$ behaviors with $3 \mathrm{P}, 4 \mathrm{P}$ and $5 \mathrm{P}$ behaviors present in small parametric spaces. However, in this region the branches are close together and consequently masked by experimental noise. From $5.96 \mathrm{~V}$ the diagram clearly indicates 2P behavior, which eventually converges to $1 \mathrm{P}$. Above $\mathrm{Vin}=7.8 \mathrm{~V}$ to $10 \mathrm{~V}$, the diagram branches into $3 \mathrm{P}$ behavior.

Many studies of DBDs have highlighted the importance of space charge accumulation in maintaining a stable discharge $[29,38]$. In theory, during each voltage half cycle, plasma forms resulting in surface charge accumulation on the surrounding dielectric surfaces, perturbing the applied field and causing the plasma to extinguish. In an ideal system, this process operates in equilibrium and all discharge events are generated and suppressed equally, yielding a periodic discharge. Numerical studies on the dynamical behavior of barrier discharges indicate that space charge accumulation is strongly influenced by the characteristics of the previous discharge event [23] and therefore may play an important role in dynamic 
evolution of plasma into a quasi-periodic or chaotic mode. Alternation between strong and weak discharge events is represented in the phase space by a limit cycle containing two loops, and as two branches on a bifurcation diagram, such as those observed in figure $3(\mathrm{~g})$. It is noted that chaotic behavior always immediately follows the gas breakdown. This is likely to be an extreme case, where the space charge balance cannot be maintained with any regularity, and the system transcends into chaotic dynamics. Recently a chaotic mode is observed to transit from a periodic mode as the input voltage increases [13], and therefore there are likely to be more than one transition routes to chaos in atmospheric plasma jets.

\subsection{Two parameter analysis}

In any plasma jet system, the gas flow rate plays a key role in defining the physical characteristics of the discharge; parameters such as gas temperature and particle diffusion are all strongly influenced by the gas flow [39, 40]. By introducing the gas flow rate as the second control parameter, it is possible to create a map of dynamical regimes in the plane of the two control parameters. Figure 4 shows a complete map of dynamical behaviors obtained by varying both the input voltage and the gas flow rate. The map consists of 4500 points, each point describing the plasma behavior at a given gas flow rate and input voltage. The procedure for acquiring experimental data involved measuring the discharge current, whilst the input voltage was increased from 1 to $10 \mathrm{~V}$ in $0.1 \mathrm{~V}$ increments at a fixed gas flow. Upon reaching $10 \mathrm{~V}$, the gas flow was incremented by $0.1 \mathrm{slm}$ and the voltage again increased from 1 to $10 \mathrm{~V}$ whilst recording the discharge current. Under each condition, the phase portrait was plotted and the stroboscopic section calculated. Each section was examined in turn to establish the dynamics of the system. Limit cycles of period one, two and three are denoted in figure 4 as $1 \mathrm{P}, 2 \mathrm{P}$ and $3 \mathrm{P}$, respectively. Quasi-periodic motion is denoted with a ' $T$ ' and regions of chaotic 
motion are marked 'Chaos'. Small parametric pockets where 3P, 4P and 5P behaviors may emerge onto the background of a dominating $2 \mathrm{P}$ behavior, such as those at $\mathrm{V}_{\text {in }}$ $=5.5-6.2 \mathrm{~V}$ in figure $3(\mathrm{~g})$, were observed and their appearance was found to be sensitively influenced by small variations in the gas flow rate or the input voltage. These are ignored in figure 4, because they occupy very small spaces in the 2D map in figure 4 and their inclusion compromises the clarity of the map.

Analysis of the map shown in figure 4 indicates that chaotic behaviors occur in a Cshaped parametric region. Irrespective of the gas flow rate, the discharge always exhibits chaotic motion after the breakdown, consistent with the analysis discussed previously. At low and high gas flow rates (above $5.8 \mathrm{slm}$ or below $1.2 \mathrm{slm}$ ), the discharge also exhibits chaotic behavior over a significant range of the input voltage. It is possible that the gas flow could upset the delicate balance of space charges through its influence on the transport of charged particles to the dielectric surface. However, when considering high gas flow rates, a more likely cause is the transition from a laminar gas flow to a turbulent gas flow. For buoyant helium jets in ambient air, it has been shown both numerically and experimentally that turbulence is present even at a low Reynolds number in conjunction with a Richardson number [41, 42]. A turbulent gas flow is by definition chaotic and the impact of a turbulent flow on the dynamic characteristics of the plasma jet is likely to be significant, not least because of the irregular mixing of the flowing helium with ambient air that dynamically changes the discharge chemistry. Indeed, for helium flow at $4 \mathrm{slm}$ exiting from a narrow tube of $1.6 \mathrm{~mm}$ inner diameter, the Reynolds number was found to be 2.64 $\times 105$ suggesting a turbulent flow. It is important to note, however, that both chaotic and periodic discharges exist at a turbulent helium rate of $4 \mathrm{slm}$ (see figure 4), suggesting that turbulence in the gas flow is unlikely to be the most important cause of chaos in our plasma jet system. 
At low flow rates, it was observed that the point, at which the plasma plume strikes the ground electrode, fluctuates considerably. This is likely a result of too little helium reaching the ground electrode to sustain a discharge, again influencing the gas composition and consequently the discharge chemistry. From the $2 \mathrm{D}$ map, it is clear that the central region, defined by moderate flow rates between 3 and $5 \mathrm{slm}$ and moderate to high input voltages between 6 and $9 \mathrm{~V}$, is where the most regular and repeatable operation occurs. Such a map could be used to preselect the optimum control parameter values for a given application, thus serving as an indirect control mechanism.

\subsection{Impact on chemistry}

It is known that the timescale of electron production and loss in a gas plasma can be very different from those of excited and reactive plasma species. For lowtemperature atmospheric plasmas in helium-oxygen species, the emission of excited atomic oxygen at $777 \mathrm{~nm}$ usually reaches its peak at the same time as the discharge current but their peak values are preceded by some $40 \mathrm{~ns}$ by the peak emission of excited helium at $706 \mathrm{~nm}$ [43]. On the other hand, the emission pulse of $0 *$ is more than three times as wide as the pulse widths of both the discharge current and the He* emission line [43]. Similar differences also exist for the $\mathrm{OH}$ emission line at 309 $\mathrm{nm}$ and $\mathrm{N} 2+$ line at $391 \mathrm{~nm}$ [43]. These suggest that dynamic behaviors in the discharge current may not be used as a reliable indicator of dynamic behaviors of excited plasma species and hence possible chaos in reaction chemistry in plasma. In addition, they confirm that optical emission signatures of excited helium and atomic oxygen may be used as an independent second observable to the first observable of the discharge current. Given these, it is of interest to examine possible chaotic behaviors in plasma chemistry by means of optical emission signatures under conditions where the discharge current exhibits clear chaotic characteristics. 
As an indicator of reaction chemistry of the plasma jet, figures 5(a) and (b) show phase space representations of the optical signature of the helium $3 s 3 S \rightarrow 2 p 3 P$ emission obtained while the discharge is operating with a flow rate of $4 \mathrm{slm}$ in the chaotic regime at $\mathrm{V}_{\text {in }}=3.5 \mathrm{~V}$, and immediately after the chaotic-periodic transition point at $3.54 \mathrm{~V}$, respectively. The phase space representations were constructed as described previously, but confined to two dimensions for the sake of clarity. It is clear that figures 5(a) and (b) are very different: figure 5(a) shows a chaotic attractor while figure $5(\mathrm{~b})$ a limit cycle of period $2 \mathrm{P}$, highlighting that the production of excited species fluctuates in a chaotic manner. Figures $5(a)$ and (b) serve to show that the amount of excited helium produced on a cycle-by-cycle basis varies chaotically when the discharge is operating in a chaotic regime. It also raises an interesting question: are other excited species produced in a chaotic manner and, if so, are the optical signatures linked so that a large current spike produces a blanket high flux of all excited species, or do the fluxes of species vary independently from one another? To answer this question we compare the line emission of helium 3s3S $\rightarrow 2 \mathrm{p} 3 \mathrm{P}(706 \mathrm{~nm})$ and atomic oxygen $3 \mathrm{p} 5 \mathrm{~S} \rightarrow 3 \mathrm{~s} 5 \mathrm{P}(777 \mathrm{~nm})$. The line ratio in the chaotic and periodic regimes is depicted in figures 5(c) and (d), respectively. Remarkably, in the chaotic regime the cycle-to-cycle line ratio of $\mathrm{He} * / \mathrm{O} *$ varies considerably, reaching a maximum of 45.78 and a minimum of 2.14 with a standard deviation of 8.81 . In comparison, the cycle-to-cycle $\mathrm{He} * / \mathrm{O} *$ line ratio in the periodic regime varies considerably less, with a standard deviation of 1.33 . This is a direct indicator that not only does the density of excited species fluctuate in a chaotic manner, but the chemical composition of the plasma also changes in a chaotic manner. Clearly, there is much scope to repeat the above investigation for other reactive plasma species including ground-state species (e.g. hydrogen oxides and ozone); however, as a whole, plasma chemistry does seem to have a chaotic regime 
even though detailed dynamic behaviors of relevant plasma species may differ from those of the discharge current.

The cycle-to-cycle variation of excited species indicates dynamic changes to key physical parameters in the discharge, including the electron energy distribution function. Such a finding has not been reported previously and is of paramount importance, given that the electron energy distribution partly dictates the plasma chemical composition-a key application driver. In the rapidly emerging field of plasma medicine, a key strength of low-temperature plasmas is believed to be the synergy among different plasma species and an ability to electrically tune such synergy by varying the operating point of the plasma [8]. This offers a vast parametric space within which to search and optimize plasma-based therapeutic solutions. Figure 5 suggests that even at a given operating point plasma chemistry may vary chaotically over a relatively large timescale of many hundred voltage cycles. Effectively, these results reveal a previously unknown dimension of varying plasma chemistry. It is worth noting that with this paper, chaos has been observed in low-temperature atmospheric plasma jets in helium mixture with both oxygen [14, 15] and water vapor [13]. It is conceivable that it may exist in atmospheric plasmas in other gas mixtures and indeed other electrode configurations.

\section{Summary}

Chaos in plasma dynamics and plasma chemistry is an important scientific topic for low-temperature atmospheric plasmas with clear and significant implication to their numerous applications. This contribution has reported experimental observations of the dynamic behavior of an atmospheric-pressure dielectric barrier plasma jet. Using the discharge current as the first observable, the input voltage and the gas flow rate are used as two independent control parameters to map out the dynamics over a wide parameter space and to identify operating conditions of 
periodic, quasi-periodic and chaotic behavior. Then employing optical emission signatures of two excited plasma species as the second observable, it is shown that the density and composition of excited plasma species generated within the jet can fluctuate in a chaotic manner; a finding that suggests cycle-to-cycle variation in key plasma parameters such as the electron temperature and hence plasma chemistry that the electron energy distribution function influences strongly. For several application areas such as processing of abiotic materials, lighting and display, chaotic behaviors in plasma dynamics may be undesirable for ensuing reproducible application performance and the 2D map of plasma dynamics in figure 4 provides a quantitative guidance to the selection of preferable plasma conditions. In the field of plasma medicine where gas discharges are used to treat cell-containing materials, chaos in plasma dynamics and plasma chemistry represents a new regime in which the issue of drug resistance may be effectively addressed. 


\section{Figure Caption:}

Figure 1: $\quad$ Schematic of the experimental apparatus.

Figure 2: $\quad$ Current and voltage traces obtained at an input voltage of (a) $3.5 \mathrm{~V}$, (b) $5.8 \mathrm{~V}$ and (c) $7.5 \mathrm{~V}$. Power spectral densities obtained at an input voltage of (d) $3.5 \mathrm{~V}$, (e) $5.8 \mathrm{~V}$ and (f) $7.5 \mathrm{~V}$.

Figure 3: Three-dimensional phase space reconstructions of the discharge current for the plasma jet operating with an input voltage of (a) 3.5 $\mathrm{V}$, (b) $5.8 \mathrm{~V}$ and (c) $7.5 \mathrm{~V}$. Stroboscopic sections shown in (d)-(f) are calculated from the phase space reconstructions in (a)-(c), respectively, and $(\mathrm{g})$ shows the one-parameter bifurcation diagram obtained when the input voltage is increased from $2.5 \mathrm{~V}$ to $10 \mathrm{~V}$. Here $\mathrm{K}$ denotes the index of excitation cycles used for stroboscopic sections.

Figure 4: $\quad$ Two-parameter map of dynamical behavior obtained by varying the input voltage and gas flow rate: $1 \mathrm{P}, 2 \mathrm{P}$ and $3 \mathrm{P}$ denote a limit cycle of period one, two and three, respectively. ' $\mathrm{T}$ ' denotes a torus (quasiperiodic behavior) and 'Chaos' denotes chaotic behavior. The white region marked ' $1 \mathrm{P}^{\prime}\left(\mathrm{V}_{\text {in }}<2.6-3.2 \mathrm{~V}\right)$ corresponds to the prebreakdown region.

Figure 5: $\quad$ Two-dimensional phase space reconstructions of the optical signal of the helium $3 \mathrm{~s} 3 \mathrm{~S} \rightarrow 2 \mathrm{p} 3 \mathrm{P}$ emission operating with an input voltage of (a) $3.5 \mathrm{~V}$ and (b) $3.54 \mathrm{~V}$. Cycle-to-cycle line ratio of helium $3 \mathrm{~s} 3 \mathrm{~S} \rightarrow$ 2 p3P/oxygen 3 p5S $\rightarrow 3$ s5P with an input voltage of (c) $3.5 \mathrm{~V}$ and (d) $3.54 \mathrm{~V}$. 


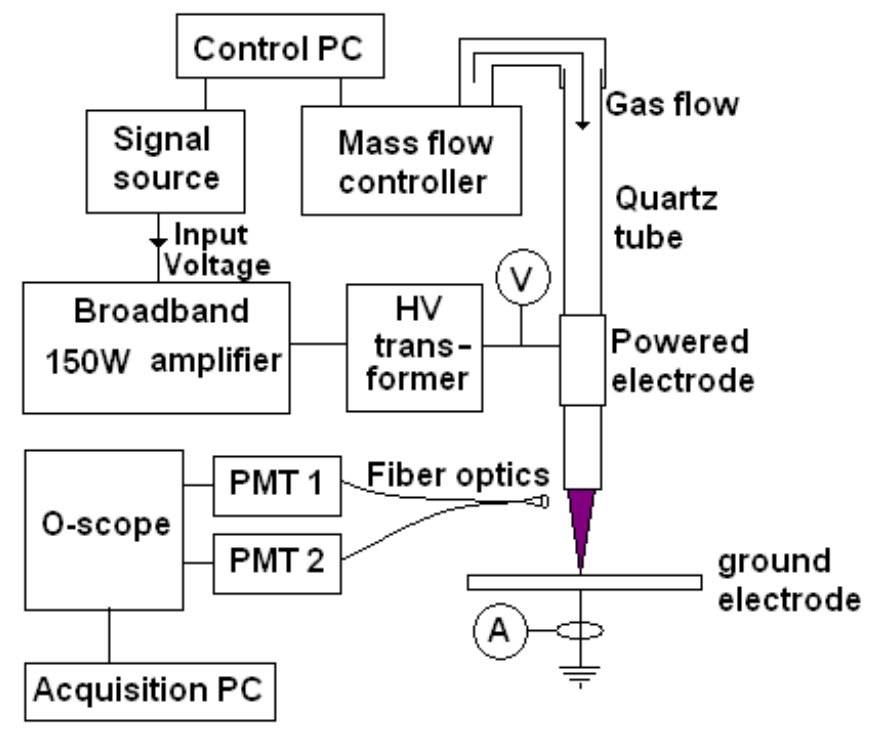

Figure 1: $\quad$ Schematic of experimental apparatus. 

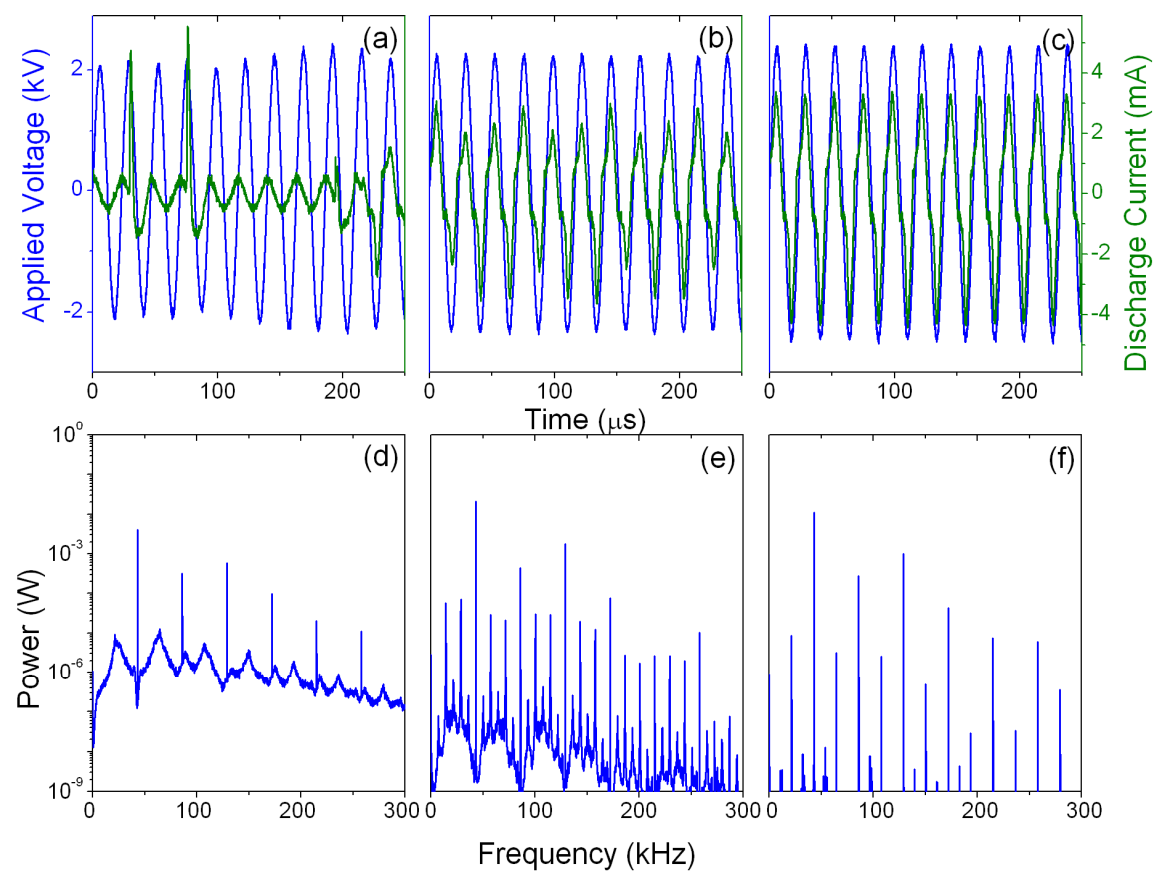

Figure 2: $\quad$ Current and Voltage traces obtained at an input voltage of (a) 3.5V, (b) $5.8 \mathrm{~V}$, and (c) $7.5 \mathrm{~V}$. Power spectral densities obtained at an input voltage of (d) $3.5 \mathrm{~V}$, (e) $5.8 \mathrm{~V}$, and (f) $7.5 \mathrm{~V}$. 

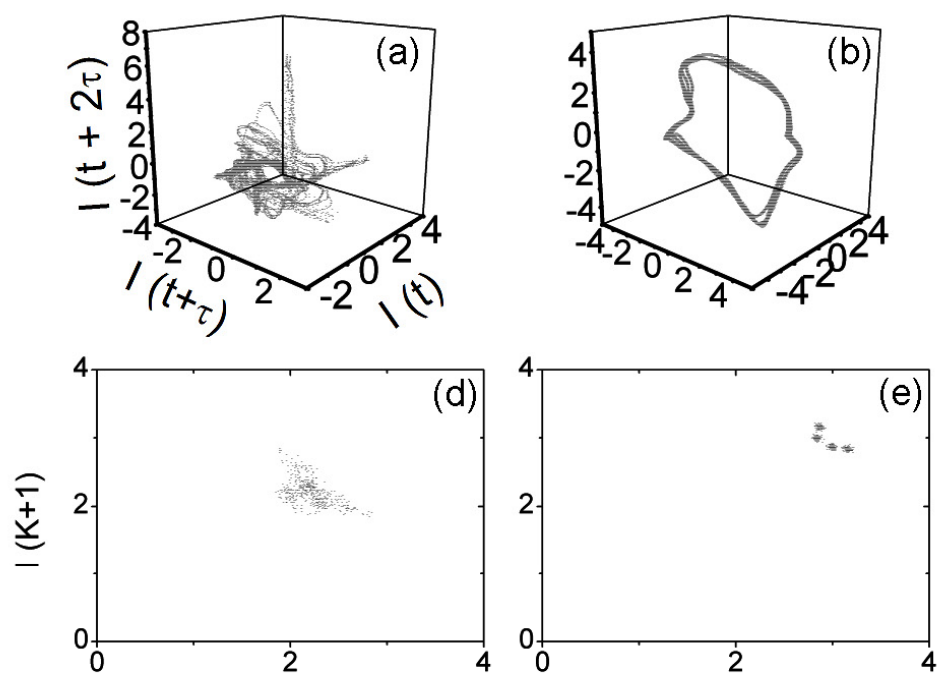

$\mathrm{I}(\mathrm{K})$

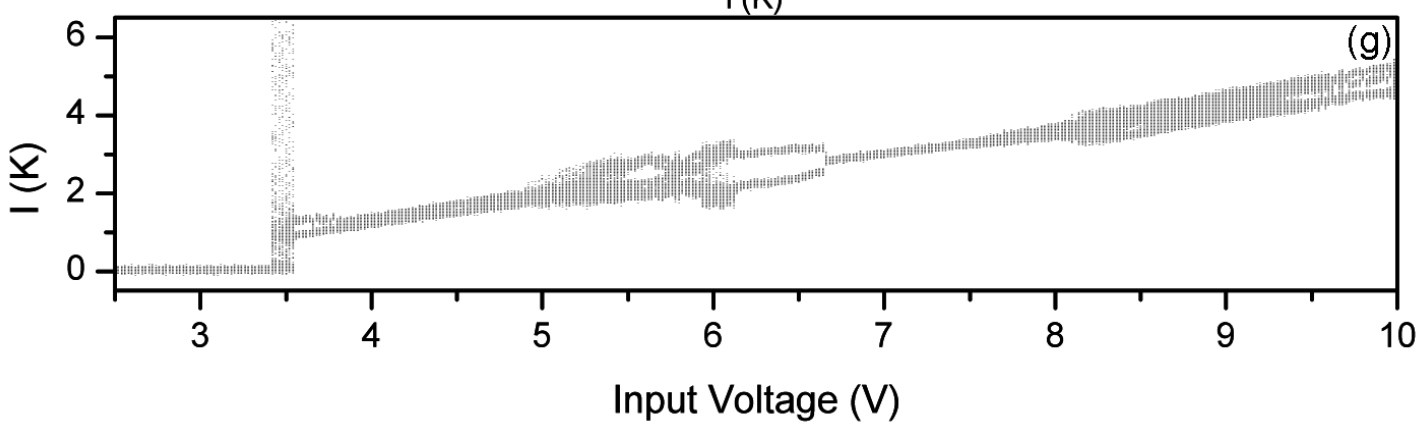

Figure 3: $\quad$ Three-dimensional phase space reconstructions for the jet operating with an input voltage of (a) $3.5 \mathrm{~V}$, (b) $5.8 \mathrm{~V}$, and (c) $7.5 \mathrm{~V}$. $5 \mathrm{~d}-5 \mathrm{f}$ show stroboscopic sections calculated from the phase space reconstructions in $5 \mathrm{a}$ to $5 \mathrm{c}$ respectively. $5 \mathrm{~g}$ shows the one-parameter bifurcation diagram obtained when the input voltage is increased from $2.5 \mathrm{~V}$ to 10V. 


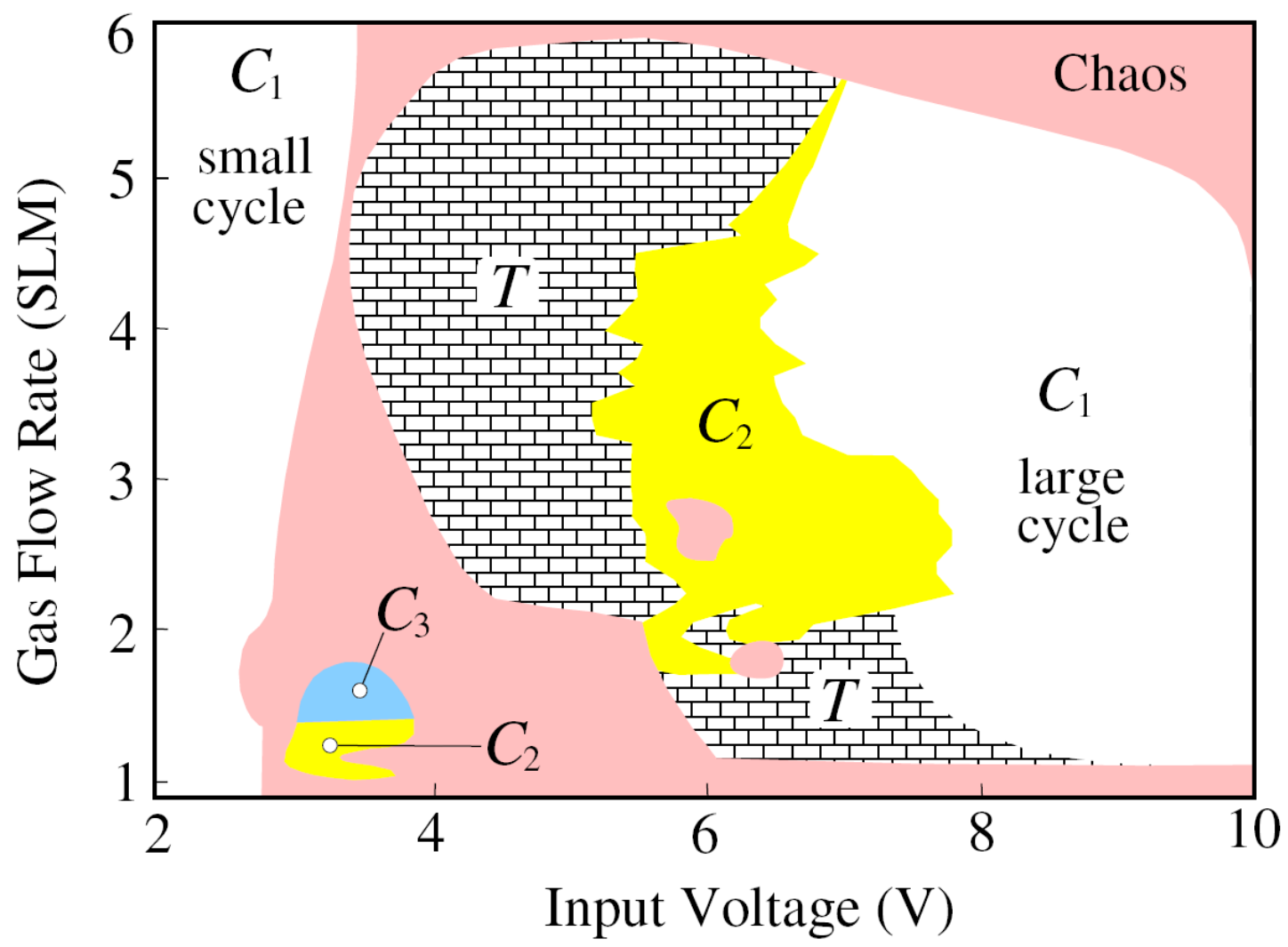

Figure 4: $\quad$ Map of 2D dynamical behavior obtained by varying the input voltage and gas flow rate: $C_{1}, C_{2}, C_{3}$ denote a limit cycle of period one, two and three respectively. $T$ denotes a torus (quasiperiodic behavior) and Chaos denotes chaotic behavior. 

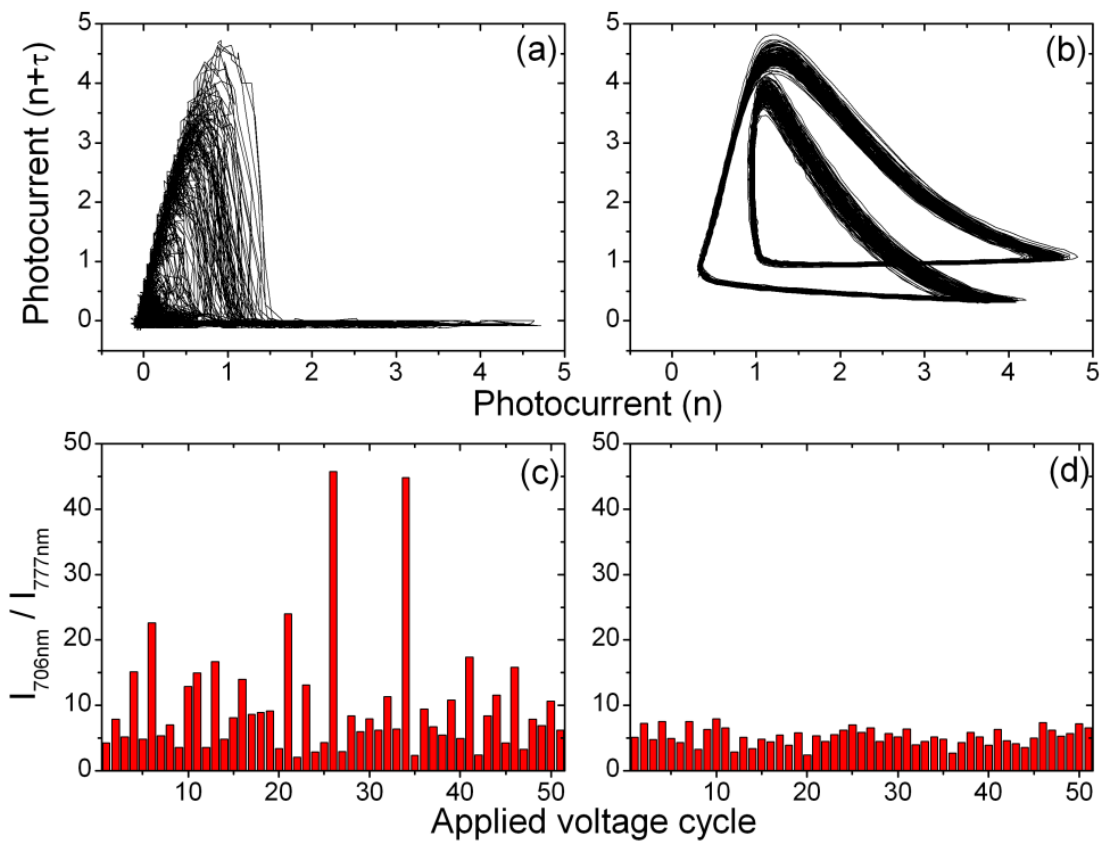

Figure 5: Two-dimensional phase space reconstructions of the helium $3 s^{3} \mathrm{~S} \rightarrow 2 \mathrm{p}^{3} \mathrm{P}$ optical signature operating with an input voltage of (a) $3.5 \mathrm{~V}$ and (b) $3.54 \mathrm{~V}$. Cycle-to-cycle line ratio of helium $3 \mathrm{~s}^{3} \mathrm{~S} \rightarrow 2 \mathrm{p}^{3} \mathrm{P} /$ oxygen $3 \mathrm{p}^{5} \mathrm{~S} \rightarrow 3 \mathrm{~s}^{5} \mathrm{P}$ with an input voltage of (c) $3.5 \mathrm{~V}$ and (d) $3.54 \mathrm{~V}$. 


\section{References}

[1] Laroussi M and Akan 2007 Plasma Process. Polym. 4777

[2] Cao Z, Nie Q, Bayliss D L, Walsh J L, Ren C S Wang D Z and Kong M G 2010 Plasma Sources Sci. Technol. 19025003

[3] Bardos L and Barankova H 2000 Surf. Coat. Technol. 133-134 522

[4] Noeske M, Degenhardt J, Strudthoff S and Lommatzsch U 2004 Int. J. Adhes. Adhes. 24171

[5] Walsh J W and Kong M G 2007 Appl. Phys. Lett. 92251504

[6] Yan Z, Stitz L, Heeg P, Pfaff E and Roth K 2004 Infection Control Hosp. Epidemiol. 25280

[7] Stoffels E, Flikweert A J, Stoffels W W and Kroesen G M W 2002 Plasma Sources Sci. Technol. 11383

[8] Kong M G, Kroesen G, Morfill G, Nosenko T, Shimizu T, van Dijk J and Zimmermann J L 2009 New J. Phys. 11115012

[9] Walsh J L, Shi J J and Kong M G 2006 Appl. Phys. Lett. 88171501

[10] Lu X P and Laroussi M 2006 J. Appl. Phys. 100063302

[11] Walsh J L and Kong M G 2008 Appl. Phys. Lett. 93111501

[12] Bruggeman P, Liu J, Degroote J, Kong M G, Vierendeels J and Leys C 2008 J. Phys. D: Appl. Phys. 41215201

[13] Liu J J and Kong M G 2011 J. Phys. D: Appl. Phys. 44345203

[14] Bing Q, Huang J, Zhang Z and Wang D 2008 Chin. Phys. Lett. 253323

[15] Walsh J L, Iza F, Janson N B, Law V J and Kong M G 2010 J Phys D: Appl. Phys 43075201

[16] Braun T, Lisboa J A and Gallas J A C 1992 Phys. Rev. Lett. 682770

[17] Weixing D, Wei H, Xiaodong W and Yu C X 1993 Phys. Rev. Lett. 70170

[18] Fukuyama T, Watanabe Y, Taniguchi K, Shirahama H and Kawai Y 2006 Phys. Rev. E 74 016401

[19] Carpinelli G, Iacovone F, Russo A and Varilone F 2004 IEEE Trans. Power Deliv. 191869

[20] Ghorui S and Das A K 2004 IEEE Trans. Plasma Sci. 32296

[21] Atipo A, Bonhomme G and Pierre T 2002 European Phys. J. D 1979

[22] Cheung P Y and Wong A Y 1987 Phys. Rev. Lett. 59551

[23] Wang Y H, Zhang Y T, Wang D Z and Kong M G 2007 Appl. Phys. Lett. 90071501

[24] Zhang J, Wang Y and Wang D Z 2010 Phys. Plasmas 17043507

[25] Boccaletti S, Grebogi C, Lai Y C, Mancini H and Maza D 2000 Phys. Rep. 329103

[26] Boeuf J P 2003 J. Phys. D: Appl. Phys. 36 R53

[27] Borcia G, Anderson C A and Brown N M D 2003 Plasma Sources Sci. Technol. 12335

[28] Kogelschatz U,Eliasson B and Egli W 1999 Pure Appl. Chem. 711819

[29] Massines F, Rabehi A, Decomps P, Gadri R B, Segur' P and Mayoux C 1998 J. Appl. Phys. 83 2950

[30] Abarbanel H D I 1996 Analysis of Observed Chaotic Data (Berlin: Springer)

[31] Ladyzhenskaya O A 1972 J. Sov. Math. 3458

[32] Mallet-Paret J 1976 J. Diff. Eqns 22331

[33] Ruelle D 1982 Ann. Math. 115243

[34] Packard N H, Crutchfield J P, Farmer J D and Shaw R S 1980 Phys. Rev. Lett. 45712 
[35] Sauer T, Yorke J A and Casdagli M 1991 J. Stat. Phys. 65579

[36] Gouesbet G, Meunier-Guttin-Cluzel S and Minard O 2003 Chaos and its Reconstruction (New York: Nova Science)

[37] Fraser A M and Swinney H L 1986 Phys. Rev. A 331134

[38] Deng X T and Kong M G 2004 IEEE Trans. Plasma Sci.32 1709

[39] Xiong Q, Lu X, Ostrikov K, Xiong Z, Xian Y, Zhou F, Zou C, Hu J, Gong W and Jiang Z 2009 Phys. Plasma 16043505

[40] Cao Z, Walsh J L and Kong MG 2009 Appl. Phys. Lett. 94021501

[41] Satti R P and Agrawal A K 2006 Int. J. Heat Fluid Flow 27336

[42] Karakas E, Koklu M and Laroussi M 2010 J. Phys. D: Appl. Phys. 43155202

[43] Walsh J L and Kong MG 2006 Appl. Phys. Lett. 89231503 